\title{
Repercussões do câncer infantil para o cuidador familiar: revisão integrativa
}

\author{
Impact of childhood cancer for family caregivers: integrative review \\ Repercusión del cáncer infantil a los cuidadores familiares: revisión integradora
}

\begin{abstract}
Daniela Doulavince Amador', Isabelle Pimentel Gomes", Altamira Pereira da Silva Reichert ${ }^{\mathrm{III}}$, Neusa Collet ${ }^{\mathrm{IV}}$

' Universidade Federal da Paraíba, Programa de Pós-Graduação em Enfermagem (Mestranda). João Pessoa-PB, Brasil.

"Universidade Federal da Paraíba, Programa de Pós-Graduação em Enfermagem (Doutoranda). João Pessoa-PB, Brasil.

I" Universidade Federal da Paraíba, Centro de Ciências da Saúde, Departamento de Enfermagem de Saúde Pública e Psiquiatria. João Pessoa-PB, Brasil.

Iv Universidade Federal da Paraíba, Centro de Ciências da Saúde, Departamento de Enfermagem de Saúde Pública e Psiquiatria, Programa de Pós-Graduação em Enfermagem. João Pessoa-PB, Brasil.
\end{abstract}

Submissão: 01-09-2011 Aprovação: 01-04-2013

\section{RESUMO}

Trata-se de uma revisão integrativa da literatura com o objetivo de identificar as repercussões do câncer infantil para o cuidador familiar. A pesquisa foi realizada nas bases de dados LILACS, PubMed, SciELO, Adolec e Cochrane, buscando-se artigos em português, inglês e espanhol, publicados entre 2005 e 2010, usando os descritores: cuidadores, criança, câncer e enfermagem. Foram obtidos dezoito artigos. A análise das publicações selecionadas permitiu a identificação de quatro categorias temáticas: sentimentos vivenciados pelo cuidador familiar; repercussões físicas e psicológicas do sofrimento do cuidador familiar; Impacto financeiro do câncer infantil na vida do cuidador e necessidade de apoio social ao cuidador familiar. Conclui-se que os prejuízos à vida do cuidador podem fragilizar a relação criança/cuidador necessitando efetiva atuação da equipe de saúde no intuito de oferecer suporte, acompanhamento e orientação.

Descritores: Cuidadores; Criança; Neoplasias; Enfermagem.

ABSTRACT

This is an integrative review that aimed to identify the repercussions of childhood cancer for the family caregiver. The research was done in the databases LILACS, PubMed, SciELO, Adolec and Cochrane, searching articles in English, Portuguese and Spanish; published between 2005 and 2010; using the descriptors: caregivers, children, cancer and nursing. Eighteen articles was found. Through the analysis four themes were identified: feelings experienced by family caregivers; physical repercussions and psychological distress of family caregivers; financial impact of childhood cancer in the caregiver's life, and need for social support to family caregivers. It was concluded that the damage to the caregiver's life can weaken the relationship between child/ caregiver, determining the need of effective performance of the health team to offer support, guidance and monitoring.

Key words: Caregivers; Child; Neoplasms; Nursing.

\section{RESUMEN}

Se trata de una revisión integradora de la literatura que tuvo el objetivo de identificar los efectos del cáncer infantil para el cuidador familiar. El estudio se llevó a cabo en las bases de datos LILACS, PubMed, SciELO, Adolec y Cochrane, buscándose artículos en portugués, inglés y español, publicados entre 2005 y 2010, con las palabras clave: cuidadores, niños, cáncer y enfermería. La búsqueda resultó en 18 artículos seleccionados. El análisis de las publicaciones seleccionadas permitió la identificación de cuatro temas: los sentimientos experimentados por los cuidadores familiares; las repercusiones físicas y psicológicas del sufrimiento de los cuidadores familiares; el impacto financiero de cáncer en la vida del cuidador y la necesidad de apoyo social para los cuidadores familiares. Se concluye que el daño a la vida del cuidador puede debilitar el vínculo niño/ cuidador que requieren una acción eficaz del equipo de salud con el fin de ofrecer apoyo, orientación y supervisión.

Palabras clave: Cuidadores; Niño; Neoplasias; Enfermería. 


\section{INTRODUÇÃO}

O processo de adoecer infantil traz consigo repercussões que fragilizam e vulnerabilizam criança e família ${ }^{(1-2)}$. Quando esse processo é representado pela sobrecarga e pelo estigma que acompanha o câncer infantil, torna-se ainda mais angustiante. Ao adentrar no universo familiar e nos diversos aspectos do contexto da criança com câncer, percebe-se que existe uma relação que fica sobremaneira evidenciada: o vínculo criança/cuidador principal.

Os cuidadores, na maioria das vezes representados pela mãe, apesar de desempenharem um papel fundamental na redução do sofrimento da criança e auxiliar no seu bem estar, são marginalizados; seu trabalho não é valorizado e não são reconhecidos como pessoas que estão passando por um processo doloroso e que precisam de ajuda, apoio e orientação ${ }^{(3)}$.

Os cuidadores de crianças com doenças crônicas possuem quatro tipos de responsabilidades: gestão da doença, que inclui cuidados com o tratamento, acompanhamento, vigilância de sinais e sintomas, resolução de problemas e tomada de decisão; manutenção da unidade familiar, que significa atender as demandas da família e, ao mesmo tempo, satisfazer as necessidades de saúde e de desenvolvimento de cada membro; manutenção pessoal, incluindo a saúde física, emocional e espiritual do cuidador; e identificação, acesso e coordenação dos recursos que envolvem a busca por serviços e profissionais de saúde para o cuidado à criança ${ }^{(4)}$.

Considerando todas as responsabilidades impostas ao cuidador, esse estudo tem o objetivo de analisar, na produção científica de profissionais da saúde, as repercussões do câncer infantil para o cuidador familiar da criança. Os resultados deste estudo buscam apresentar uma atualização sobre a temática e indicar subsídios aos profissionais de enfermagem para a produção do cuidado estendido à família da criança com câncer.

\section{METODOLOGIA}

Trata-se de uma revisão integrativa da literatura. As seguintes etapas foram percorridas: identificação do tema e formulação da questão norteadora; estabelecimento de critérios para inclusão e exclusão de estudos; definição das informações a serem extraídas dos estudos selecionados; avaliação dos estudos incluídos na revisão integrativa; categorização dos estudos; interpretação dos resultados; apresentação da revisão.

Considerando-se que o câncer infantil pode trazer importantes consequências ao cuidador principal familiar e que na prática profissional o foco de atenção, quase sempre, é a criança, cabendo ao cuidador uma localização mais à margem dos acontecimentos, a questão norteadora foi: Quais as repercussões do câncer infantil para o cuidador principal da criança?

A partir do questionamento, procedeu-se a coleta nas bases de dados LILACS, PubMed; SciELO; Adolec e Cochrane, estabelecendo-se os seguintes critérios de inclusão: artigos que abordassem a temática do cuidador familiar da criança com câncer, que subsidiassem a assistência de enfermagem; artigos em inglês, português e espanhol; publicados entre os anos de 2005 e 2010. Excluíu-se os artigos que apontassem o profissional de saúde como cuidador.

A pesquisa foi realizada utilizando-se os descritores DECS/ MESH: cuidadores (caregiver), criança (child), câncer (cancer), enfermagem (nursing), realizando-se cruzamentos triplos e/ou quádruplos. Os dados foram coletados de agosto a outubro de 2010. Após a leitura dos títulos e resumos, os estudos selecionados foram analisados com auxílio de um instrumento já validado, avaliando-se dados referentes ao periódico, autor, estudo e o nível de evidência ${ }^{(5)}: 1$ - revisões sistemáticas ou metanálise de relevantes ensaios clínicos; 2 - evidências de pelo menos um ensaio clínico randomizado controlado bem delineado; 3 - ensaios clínicos bem delineados sem randomização; 4 - estudos de coorte e de caso-controle bem delineados; 5 - revisão sistemática de estudos descritivos e qualitativos; 6 - evidências derivadas de um único estudo descritivo ou qualitativo; 7 - opinião de autoridades ou comitês de especialistas.

A síntese dos descritores utilizados, das bases de dados e das referências selecionadas está descrita no quadro a seguir:

\begin{tabular}{|c|c|c|c|}
\hline $\begin{array}{c}\text { Base de } \\
\text { dados }\end{array}$ & Descritores cruzados & $\begin{array}{c}\text { Referências } \\
\text { obtidas }\end{array}$ & $\begin{array}{c}\text { Referências } \\
\text { selecionadas }\end{array}$ \\
\hline PUBMED & Caregiver/child/cancer/nursing & 75 & 7 \\
\hline LILACS & Cuidadores/criança/câncer/enfermagem & 7 & 3 \\
\hline SCIELO & Cuidadores/criança/câncer & 3 & 3 \\
\hline ADOLEC & Cuidadores/criança/câncer & 14 & 2 \\
\hline COCHRANE & Caregiver/child/cancer & 11 & 1 \\
\hline LILACS & Cuidadores/criança/câncer & 18 & 2 \\
\hline
\end{tabular}

Quadro 1 - Distribuição das referências obtidas nas bases de dados Pubmed, Lilacs, Scielo, Adolec e Cochrane, segundo os descritores estabelecidos.

Após um refinamento dos artigos no intervalo de 2005 a 2010, e excluídos os que não correspondiam ao objeto de estudo ou que se encontravam em duplicidade com artigos já selecionados, obteve-se 18 artigos que contemplavam a questão norteadora do estudo.

\section{RESULTADOS}

Os 18 artigos selecionados estavam distribuídos em periódicos distintos, não havendo grande concentração de publicação sobre cuidadores de crianças com câncer em periódicos específicos.

A maioria dos artigos selecionados foram publicados por enfermeiros, totalizando 11 artigos $^{(3,6-15)}$; os demais eram publicações da área médica ${ }^{(16-19)}$ e de psicologia ${ }^{(20-22)}$, sendo um desses em associação com fisioterapia ${ }^{(20)}$. Parece que os pesquisadores da área de enfermagem têm se preocupado mais em publicar assuntos relacionados ao cuidador familiar, inferindo-se que há inquietação em investigar, compreender e 
assistir a criança no contexto familiar. Em relação ao idioma, 10 artigos $^{(6,8-10,13,16-19,22)}$ encontravam-se em inglês.

Os estudos foram ainda observados em relação à abordagem da pesquisa e, assim, classificados quanto ao nível de evidência. A abordagem qualitativa foi utilizada em 5 artigos, 9 abordagem quantitativa e 4 quanti-qualitativos. Entre aqueles com delineamento quantitativo, apenas um foi estudo clínico randomizado, o qual possui nível de evidência forte segundo a classificação utilizada(5). A listagem dos artigos incluídos, bem como as características citadas encontra-se no Quadro 2.

A análise das publicações selecionadas permitiu a identificação de diversas abordagens no que diz respeito às implicações do câncer infantil para o cuidador familiar. A partir das semelhanças entre os temas encontrados os resultados foram agrupados e apresentados em quatro categorias temáticas, a saber: sentimentos vivenciados pelo cuidador familiar; repercussões físicas e psicológicas do sofrimento do cuidador familiar; impacto financeiro do câncer infantil na vida do cuidador e necessidade de apoio social ao cuidador familiar.

\section{Sentimentos vivenciados pelo cuidador familiar}

Estudo realizado em um ambulatório de oncologia pediátrica evidenciou que na vivência afetiva dos cuidadores frente ao adoecimento da criança, a fase do diagnóstico e início do tratamento foram as mais difíceis, sendo estas acompanhadas de intenso sofrimento e dor ${ }^{(21)}$. Além do sofrimento emocional diante do impacto do diagnóstico, os pais de uma criança com câncer referiram ter dificuldades para impor limites no comportamento da criança, o que acabava resultando em superproteção para a criança e angústia para os pais ${ }^{(13)}$.

Um estudo realizado com pais e mães islandeses cuidadores de crianças com câncer demonstrou que as mães se sentem angustiadas por terem que coordenar, organizar e gerir serviços e recursos para toda a família e concomitantemente subsidiar suporte emocional à criança com câncer e aos demais membros. Elas também relataram que os cuidados pessoais à criança exigem muito tempo. Os pais demonstraram angústia por terem que balizar a assistência à criança, atividades no trabalho e apoio emocional ao cônjuge ${ }^{(9)}$.

\begin{tabular}{|c|c|c|c|c|c|}
\hline Base & Ano & Idioma & Área & Delineamento do estudo & Evidência $^{(4)}$ \\
\hline PUBMED & $2008^{(7)}$ & Port & Enfermagem & Quali & 6 \\
\hline PUBMED & $2009^{(6)}$ & Ing & Enfermagem & Quanti - descritivo & 6 \\
\hline PUBMED & $2008^{(16)}$ & Ing & Médico & Quanti -descritivo transversal & 6 \\
\hline PUBMED & $2005^{(8)}$ & Ing & Enfermagem & Quali & 6 \\
\hline PUBMED & $2005^{(17)}$ & Ing & Médico & Quanti -descritivo transversal & 6 \\
\hline PUBMED & $2005^{(9)}$ & Ing & Enfermagem & Quanti - descritivo longitudinal & 6 \\
\hline PUBMED & $2005^{(10)}$ & Ing & Enfermagem & Quanti - descritivo longitudinal & 6 \\
\hline LILACS & $2009^{(11)}$ & Esp & Enfermagem & Quali & 6 \\
\hline LILACS & $2007^{(3)}$ & Port & Enfermagem & Quali-Quanti descritivo transversal & 6 \\
\hline LILACS & $2009^{(12)}$ & Port & Enfermagem & Quali - descritivo exploratório & 6 \\
\hline LILACS & $2008^{(20)}$ & Port & Psicologia/ Fisioterapia & Quali & 6 \\
\hline LILACS & $2005^{(13)}$ & Ing & Enfermagem & Quali & 6 \\
\hline SCIELO & $2007^{(14)}$ & Port & Enfermagem & Quanti descritivo comparativo transversal & 6 \\
\hline SCIELO & $2009^{(15)}$ & Port & Enfermagem & Quanti-Quali & 6 \\
\hline SCIELO & $2010^{(21)}$ & Port & Psiclogia & Quanti-Quali & 6 \\
\hline ADOLEC & $2009^{(18)}$ & Ing & Médica & Quanti - transversal & 6 \\
\hline ADOLEC & $2006^{(19)}$ & Ing & Médica & Quanti: retrospectivo, tranversal & 6 \\
\hline COCHRANE & $2005^{(22)}$ & Ing & Psicologia & Ensaio Clínico Randomizado & 2 \\
\hline
\end{tabular}

Legenda: Port: Português; Ing: Inglês; Esp: Espanhol; Quanti: quantitativo; Quali: qualitativo.

Quadro 2 - Distribuição das referências incluídas na revisão integrativa, de acordo com as bases de dados, ano, idioma, área de atuação, delineamento do estudo e nível de evidência. 
O câncer infantil afeta diversas áreas da vida do cuidador, pois abre-se mão do trabalho, estudo, das horas de sono, da vida social, do lazer, prazer, da vida familiar e do cuidado pessoal $^{3}$. As mães referem que o cuidado do filho com câncer consome suas vidas em tempo integral, principalmente quando o cuidado é domicíliar, pois elas assumem a administração de medicamentos e observação de efeitos colaterais, podendo proporcionar frustração por não terem domínio na realização dessas ações, somando-se às atividades no cuidado à criança e toda a família ${ }^{(8)}$.

A percepção do cuidador sobre o cuidar da criança com câncer e neutropênica é demarcada pelas mudanças que ocorrem em diferentes esferas: ambiente, pessoas e relações; e permeada por incertezas, medo, desesperança e instabilidade. Por outro lado, essas mudanças proporcionam aprendizado, fé, autoconfiança, dedicação e autoconhecimento ${ }^{(15)}$.

A existência de preconceitos ligados à doença é um fator desencadeador de sofrimento e, nesse sentido, a percepção e conscientização da possibilidade de morte da criança pode facilitar desestruturações pessoais no cotidiano do cuidador $^{(21)}$. Os sentimentos relacionados com a religiosidade são evidenciados nos cuidadores de crianças com câncer, os quais se manifestam pelo aumento da fé e entrega da doença à vontade de Deus ${ }^{(21,7,15)}$. Os não praticantes de religião aceitaram mais a responsabilidade para si, quando comparados aos praticantes de religião que consideravam o adoecer como providência divina $^{(21)}$.

Os sentimentos do cuidador familiar da criança influenciam na sua percepção em relação à saúde e o bem estar das crianças. Foi o que demonstrou um estudo ${ }^{(10)}$ que avaliou as mudanças nas necessidades de cuidado e bem estar de mães e pais e a percepção em relação à saúde dos filhos durante o período de um ano. Revelou-se que os que não atingiram um nível significativo de bem estar tinham uma percepção mais negativa da saúde do filho. Este resultado reforça a necessidade de o cuidado ser centrado na criança e em sua família.

\section{Repercussões físicas e psicológicas do sofrimento do cui- dador familiar}

Cuidar de uma criança com câncer exige tempo e dedicação, e o esforço dispensado para esse cuidado reflete diretamente na condição de saúde do cuidador, implicando muitas vezes em sérios prejuízos físicos e psicológicos. Estudos ${ }^{(16-17)}$ de caso controle com mães que cuidavam de crianças com câncer e com as de crianças sadias apontaram que a qualidade de vida encontrava-se alterada nas mães que vivenciavam o câncer infantil. Além de alterações na saúde mental e um maior risco de desenvolvimento de depressão, essas cuidadoras tiveram a alimentação e os hábitos de sono completamente alterados.

As mudanças impostas pelo câncer e as preocupações que envolvem o universo do cuidador refletem em desgaste físico e mental, não só pelas atividades desempenhadas e pelo tempo despendido nessas atividades, mas também pelo estigma da doença. O diagnóstico de 'tensão devido ao papel de cuidador' esteve presente em $78 \%$ dos 50 cuidadores entrevistados de estudo realizado em um hospital infantil onco-hematológico $^{(14)}$. Fatores como a duração do cuidado, a preocupação com a desorganização na estrutura familiar e o fato de não aceitar que outra pessoa assuma o seu papel, são determinantes nas implicações para a saúde de quem cuida, podendo gerar complicações como hipertensão, cefaléia e dor no corpo, gastrite, depressão, entre outros. Neste estudo todos os cuidadores apresentavam alto risco para 'tensão devido ao papel de cuidador'. Os autores enfatizam o enfermeiro como essencial para diagnosticar e amenizar os fatores de risco a que esses cuidadores estão expostos.

Estudo $^{(21)}$ realizado com vinte cuidadores de crianças com leucemia apontou que $50 \%$ desses apresentavam manifestações de estresse, destacando ainda que $90 \%$ da sintomatologia apresentada por eles é de ordem psicológica. Os autores ressaltam que a percepção e conscientização da possibilidade de morte facilitam a desestruturação pessoal e manifestações de estresse. Essa sintomatologia pode influenciar negativamente na qualidade de vida dos cuidadores e na capacidade de oferecer acolhimento e responder às demandas da criança, podendo prejudicar o enfrentamento destas à situação. Outro estudo $^{(18)}$ aponta que a maioria dos cuidadores de crianças com câncer, apresentou risco psicossocial que manteve-se o mesmo quando avaliado no momento do diagnóstico e após quatro meses. Evidenciou-se que durante esse período houve um aumento significativo de sintomas de estresse traumático.

Em contrapartida, os sintomas de estresse que atingem os cuidadores podem ser em grande parte consequência de crenças negativas que, quando desmistificadas desde o início do diagnóstico, podem influenciar positivamente na trajetória do cuidador. É o que demonstra um ensaio clínico randomizado ${ }^{(22)}$ realizado com 38 cuidadores de crianças com câncer, no qual o grupo que participou do programa de intervenção que auxiliava na compreensão e no enfrentamento do diagnóstico do câncer infantil apresentou diminuição dos sintomas de ansiedade e de estresse pós-traumático, quando comparados ao grupo controle.

Impacto financeiro do câncer infantil na vida do cuidador

Dentre as inúmeras implicações que o câncer infantil traz aos cuidadores familiares, vários estudos ${ }^{(3,7,9,13,19)}$ destacam as alterações financeiras. Um deles ${ }^{(19)}$ avaliou o impacto que o câncer acarreta para os pais em relação ao aspecto financeiro, familiar/social, tensão pessoal e os mecanismos de enfrentamento. Dentre esses, o de maior relevância para os cuidadores foi o impacto financeiro, no qual $74 \%$ dos pais relataram um alto ou moderado grau de dificuldades econômicas após o diagnóstico do câncer na criança. Além de todas as despesas referentes ao tratamento e outras adicionais, a perda financeira ocorre também pelo fato de pelo menos um dos pais sair do emprego para se dedicar integralmente ao filho.

A realidade de pais e mães no cuidado à criança com câncer geralmente difere quando se trata de perda financeira, abandono ao trabalho e às atividades cotidianas. Um estudo ${ }^{(9)}$ realizado com familiares islandeses demonstrou que todos os pais trabalhavam em tempo integral, enquanto as mães, em sua maioria $(87 \%)$, encontravam-se trabalhando em tempo parcial ou não trabalhavam. Esse contexto condiz com a 
realidade da maioria das famílias na qual a mãe exerce o papel de cuidadora e o pai de provedor.

O medo de perder o emprego e o fato de não poder mais ajudar na renda familiar, no momento em que os gastos da família aumentam muito representam uma das principais fontes de preocupação do cuidador ${ }^{(3,7,13)}$.

\section{Necessidade de apoio social ao cuidador familiar}

O diagnóstico de câncer para as mães cuidadoras implica na necessidade de apoio que lhes permita orientar-se ao longo do caminho, e manter o animo e a esperança nessa difícil realidade. As mães referem necessidade de apoio informativo, espiritual, emocional e material. No entanto, os profissionais de saúde negligenciam informações e os amigos e familiares se distanciam durante essa vivência ${ }^{(11)}$. Alguns estudos referem a fragilidade da rede de apoio social dessas cuidadoras, quando evidenciam que o apoio recebido tende a diminuir ao longo do tempo desde o momento do diagnóstico ${ }^{(13,18)}$. Cuidadores familiares de crianças com câncer elucidaram ${ }^{(12)}$ que a preocupação da equipe de saúde está direcionada para o atendimento exclusivo à criança.

Cuidadores familiares de crianças com câncer que realizaram transplante de células tronco hematopoiéticas referiram que um dos maiores problemas vivenciados foi falta de informação. Alguns temas evidenciados pelos cuidadores para serem debatidos e esclarecidos foram o manejo do impacto emocional e social e as estratégias práticas para o cuidado à criança e a si próprio $^{(6)}$. Em outro estudo, ${ }^{(15)}$ a necessidade de orientação também foi citada e os cuidadores referiram déficit de informação em relação aos cuidados diários, administração de medicamentos, enfrentamento das intercorrências e o suporte afetivo.

O enfrentamento do diagnóstico e de todas as nuanças do câncer infantil depende de diversos fatores, sendo a cognição individual dos cuidadores um deles. O esclarecimento sobre os diversos aspectos da doença permite uma melhor elaboração dos sujeitos acerca de seus problemas e, para tanto, é necessário que o diálogo alcance a compreensão daqueles que cuidam. Um estudo ${ }^{(20)}$ apontou que os cuidadores de crianças com câncer que tinham maior escolaridade apresentavam melhor entendimento sobre os diversos aspectos do câncer. Por outro lado, aqueles com menor escolaridade mostravam-se apreensivos e com desconhecimento em relação ao tratamento. Os argumentos dos sujeitos da pesquisa referiam-se a carência de diálogo com os médicos, mas, de acordo com os autores $^{(20)}$, o diálogo possivelmente existia, no entanto, não era compreendido pelos familiares.

\section{DISCUSSÃO}

Apesar de grande parte dos estudos apresentarem baixo nível de evidência, eles trazem contribuições significativas sobre as repercussões do câncer infantil para o cuidador familiar. Os sentimentos vivenciados pelos cuidadores são reflexos do ônus que as atividades e os cuidados diários ininterruptos acarretam $^{(23)}$. O papel dos cuidadores nessa vivência é complexo, envolve sentimentos negativos, como angústia, decorrente da dor e do impacto que o diagnóstico traz, além da sobrecarga física e psicossocial que a família precisa suportar.

O cuidador mesmo percebendo-se fragilizado, tenta reunir forças para apoiar a criança ${ }^{(24)}$, bem como toda sua família. $\mathrm{O}$ ser que cuida é envolto em uma diversidade de sentimentos divergentes, algumas vezes, levam a pensar em desistir, mas por outro lado têm que ser a "mola" que impulsiona os demais membros.

O vínculo confere segurança aos usuários, permitindo que os trabalhadores do serviço os conheçam para melhor estabelecer as prioridades na atenção à sua saúde ${ }^{(25)}$, para isso, a compreensão da complexidade e das repercussões do cuidar precisam influenciar a percepção da equipe de saúde para a construção da atenção singular e integral. O enfermeiro deve estabelecer um elo com a díade criança-família identificando e respeitando os sentimentos vivenciados pelo cuidador, para auxiliar na superação das fragilidades e vulnerabilidades enfrentadas, fortalecendo ações que contribuem positivamente para o enfrentamento da situação.

As manifestações psicossomáticas e emocionais são decorrentes do acúmulo de atividades e da exacerbação de sentimentos vivenciados pelo cuidador ${ }^{(23)}$. Para atender a essas demandas, urge que a enfermagem supere a organização de seu processo de trabalho centrado na doença para aquele centrado na criança e na sua família, buscando a integralidade do cuidado. O fortalecimento da família e as ações de promoção da saúde direcionadas ao cuidador familiar podem contribuir para que essa vivência traumática seja minimizada.

A falta de sistema de apoio aos cuidadores que necessitam abdicar do emprego em prol dos cuidados à criança representa uma das fragilidades do sistema de saúde. O financiamento público, ainda limitado, garante apenas benefícios que auxiliam, mas não solucionam o impacto financeiro acarretado pelo câncer. Além disso, as dificuldades no provimento de recursos necessários significam o aumento da sobrecarga emocional do cuidador que vivencia também essa responsabilidade ${ }^{(2)}$. Auxiliar a família no enfrentamento desse tipo de dificuldade não implica unicamente no provimento de recursos, mas também na orientação sobre os benefícios que Ihes são de direito e no direcionamento sobre as formas de obtê-los. Lidar com a necessidade de apoio financeiro corresponde a um dos aspectos difíceis de ser vivenciado pelo enfermeiro, pois pode perceber-se incapaz diante da situação.

Assim, o diálogo e o compartilhamento de informações podem ser importantes aliados na desmistificação do câncer e na construção de novas representações pelos cuidadores, mas é imprescindível que os profissionais tenham consciência de que mais importante do que a quantidade de informação é a qualidade, a compreensão e a acessibilidade às mesmas.

Durante um diálogo é indispensável o reconhecimento do outro como um interlocutor com questionamento digno de resposta, na qual se julga possível a troca de experiências. Se há diálogo é porque ambas as partes aceitam que não há posse de uma compreensão suficiente e, enquanto houver um nível superior de entendimento a alcançar, haverá diálogo(26). Entretanto, muitas vezes o diálogo é interceptado, frágil e as ações de apoio à família, bem como ao cuidador, são limitadas e pouco efetivas. 
Incluir o familiar no plano de cuidados implica proporcionar que os mesmos se sintam competentes para o cuidado, por meio do compartilhamento do conhecimento e das habilidades necessárias para a tomada de decisão. Esse direcionamento do cuidado promove o empoderamento da família e do cuidador que se percebe sujeito ativo e participativo no cuidado à criança.

A família da criança com câncer e o seu cuidador encontram-se fragilizados e com diversas necessidades ao longo do processo da doença. Por isso, estar atento a essas necessidades é fundamental aos profissionais de saúde, com destaque para o enfermeiro, que poderá direcionar o seu cuidado de forma mais efetiva. $\mathrm{O}$ apoio social é um recurso que pode auxiliar a família e o cuidador, e a identificação da rede social é fundamental para balizar esse cuidado(23).

\section{CONCLUSÃO}

A revisão integrativa possibilitou a identificação de diversas repercussões do câncer infantil para o cuidador familiar que oferecem subsídios para o cuidado de enfermagem. Partindo-se da classificação dos níveis de evidência ${ }^{(4)}$, dezessete artigos selecionados possuem níveis de evidência fracos por se tratar de pesquisas qualitativas e quantitativas descritivas. No entanto, os resultados das publicações encontradas mostraram-se fortes o suficiente para identificar as fragilidades, necessidades e as consequências do câncer infantil para o cuidador, contribuindo para que o enfermeiro reflita sobre seu modo de produzir o cuidado à criança com câncer e sua família.

Os prejuízos à vida do cuidador, sejam emocionais, físicos ou psicológicos, podem fragilizar a relação criança/cuidador, necessitando, portanto, de efetiva atuação da equipe de saúde no intuito de oferecer suporte, acompanhamento e orientação para que o cuidador familiar sinta-se acolhido e assistido por todos os membros da equipe.

Identificar os aspectos da vida do cuidador afetados pelo câncer infantil auxilia o enfermeiro a contribuir substancialmente para que esse momento seja vivenciado de forma menos traumática. Foram apontados os sentimentos, as repercussões físicas e psicológicas de sofrimento, as necessidades de apoio social e o impacto financeiro. Embora houvesse considerações sobre as alterações familiares decorrentes do câncer infantil, não foi identificado estudo que analisasse em profundidade a estruturação ou desestruturação familiar como uma das implicações do câncer infantil para o cuidador. Para ampliar a perspectiva do cuidado estendido à família é necessário que se compreenda, também, a partir da perspectiva do cuidador, as mudanças ocorridas na interação e no funcionamento familiar.

\section{REFERÊNCIAS}

1. Leite MF, Collet N, Gomes IP, Kumamoto LHMCC. Coping with chronic condition in childhood by family caregivers: a qualitative research. Online Braz J Nurs [periódico na internet] 2010 [acesso em 30 maio 2011];9(3). Disponível em: http://www.objnursing.uff.br/index.php/ nursing/article/view/3147

2. Sitaresmi MN, Mostert S, Purwanto I, Gundy CM, Sutaryo, Veerman AJ. Chemotherapy-related side effects in childhood acute lymphoblastic leukemia in Indonesia: parental perceptions. J Pediatr Oncol Nurs 2009;26(4):198-207.

3. Beck ARM, Lopes MHBM. Cuidadores de crianças com câncer: aspectos da vida afetados pela atividade de cuidador. Rev Bras Enferm 2007;60(6):670-5.

4. Sullivan-Bolyai S, Sadler L, Knafl KA, Gilliss CL. Great expectations: A position description for parents as caregivers : Part 1. Pediatr Nurs 2003;29(6):457-61.

5. Melnyk BM, Fineout-Overholt E. Evidence-based practice in nursing e healthcare: A guide to best practice. Philadelphia: Lippincott Williams e Wilkins; 2005.

6. Mayer DK, Tighiouart H, Terrin N, Stewart S, Peterson E, Jeruss $S$, et al. A Brief report of caregiver needs and resource utilization during pediatric hematopoietic stem cell transplantation. J Pediatr Oncol Nurs 2009;26(4):223-9.

7. Comaru NRC, Monteiro ARM. O cuidado domiciliar à criança em quimioterapia na perspectiva do cuidador familiar. Rev Gaúch Enferm 2008;29(3):423-30.

8. Clarke JN, Fletcher PC, Schneider MA. Mothers' home health care work when their children have cancer. J Pediatr Oncol Nurs 2005;22(6):365-373.

9. Svavarsdottir EK. Caring for a child with cancer: a longitudinal perspective. J Adv Nurs 2005;50(2):153-61.

10. Svavarsdottir EK. Surviving childhood cancer: parents' perceptions of their child's health. J Pediatr Oncol Nurs $2005 ; 22(2): 80-8$.

11. Cacante CJV, Arias Valencia MM. Tocar los corazones en busca de apoyo: el caso de las familias de los niños con cáncer. Invest Educ Enferm 2009;27(2):170-80.

12. Wegner W, Pedro ENR. Concepções de saúde sob a ótica de mulheres cuidadoras-leigas, acompanhantes de crianças hospitalizadas. Rev Latino-Am Enferm 2009;17(1):88-93.

13. Herman ARS, Miyazaki MCOS. Intervenção psicoeducacional em cuidador de criança com câncer: relato de caso. Arq Ciênc Saúde 2007;14(4):238-44.

14. Beck ARM, Lopes MHBM. Tensão devido ao papel de cuidador entre cuidadores de crianças com câncer. Rev Bras Enferm 2007;60(5): 513-18.

15. Gelesson DD, Hiraishi LY, Ribeiro LA, Pereira SR, Gutiérrez MGR, De Domenico EBL. Significado da neutropenia e necessidades de cuidado em domicílio para os cuidadores de crianças com câncer. Rev Latino-Am Enferm 2009;17(6):933-9.

16. Klassen AF, Klaassen R, Dix D, Pritchard S, Yanofsky R, O'Donnell $M$, et al. Impact of caring for a child with 
cancer on parents' health-related quality of life. J Clin Oncol 2008;26(36):5884-9.

17. Yamazaki S, Sokejima S, Mizoue T, Eboshida A, Fukuhara S. Health-related quality of life of mothers of children with leukemia in Japan. Qual Life Res 2005;14(4):1079-85.

18. Alderfer MA, Mougianis I, Barakat LP, Beele D, DiTaranto S, Hwang WT, et al. Family psychosocial risk, distress, and service utilization in pediatric cancer: predictive validity of the Psychosocial Assessment Tool. Cancer 2009;115(18 suppl):4339-49.

19. Heath JA, Lintuuran RM, Rigguto G, Tokatlian N, McCarthy M. Childhood cancer: its impact and financial costs for australian families. Pediatr Hematol Oncol 2006;23(5):439-48.

20. Malta JDS, Schall VT, Modena CM. Câncer pediátrico: o olhar da família/cuidadores. Pediatric modern 2008; 44(3):114-8.

21. Del Bianco Faria AM, Cardoso CL. Aspectos psicossociais de acompanhantes cuidadores de crianças com câncer: stress e enfrentamento. Estud Psicol (Campinas) 2010;27(1)13-20.
22. Kazak AE, Simms S, Alderfer MA, Rourke MT, Crump T, McClure $\mathrm{K}$, et al. Feasibility and preliminary outcomes from a pilot study of a brief psychological intervention for families of children newly diagnosed with cancer. J Pediatr Psychol 2005;30(8):644-55.

23. Di Primio AO, Schwartz E, Bielemann VLM, Burille A, Zillmer JGV, Feijó AM. Rede social e vínculos apoiadores das famílias de crianças com câncer. Texto \& Contexto Enferm 2010;19(2):334-42.

24. Bortolote GS, Brêtas JRS. O ambiente estimulador ao desenvolvimento da criança hospitalizada. Rev Esc Enferm USP 2008;42(3):422-9.

25. Queiroz MVO, Ribeiro EMV, Pennafort VPS. Assistência ao adolescente em um serviço terciário: acesso, acolhimento e satisfação na produção do cuidado. Texto \& Contexto Enferm 2010;19(2):291-9.

26. Ayres JRCM. Cuidado: trabalho e interação nas práticas de saúde. In: Para compreender o sentido prático das ações de saúde: contribuições da hermenêutica filosófica. Rio de Janeiro: Abrasco; 2009. p.284. 